九州大学学術情報リポジトリ

Kyushu University Institutional Repository

\title{
BIAS IN THE PETO ONE-STEP ESTIMATOR FOR THE COMMON ODDS RATIO
}

Sato, Toshiya

Department of Biostatistics, Kyoto University, School of Public Health

https://doi.org/10.5109/12587

出版情報: Bulletin of informatics and cybernetics. 37, pp.13-18，2005-12. Research Association of Statistical Sciences

バージョン :

権利関係 : 


\section{BIAS IN THE PETO ONE-STEP ESTIMATOR FOR THE COMMON}

ODDS RATIO

by

Tosiya SATo

Reprinted from the Bulletin of Informatics and Cybernetics

Research Association of Statistical Sciences, Vol.37

FUKUOKA, JAPAN

2005 


\title{
BIAS IN THE PETO ONE-STEP ESTIMATOR FOR THE COMMON ODDS RATIO
}

\author{
By
}

Tosiya SAто*

\begin{abstract}
The one-step method in the estimation of the common odds ratio proposed by Peto was used to summarize the results from meta-analysis. For some unbalanced data, it was known to have severe upward (non-null) asymptotic bias. In this paper, the asymptotic expectation of the Peto one-step estimator is derived and its bias is evaluated numerically over 1152 parameter combinations.
\end{abstract}

Key Words and Phrases: asymptotic bias, meta-analysis, the Peto one-step estimator.

\section{Introduction}

The Peto one-step method is frequently used to estimate a summary odds ratio for meta-analysis of clinical trials (Yusuf et al., 1985; Mosteller and Chalmers, 1992; Petitti, 1994). It is based on a homogeneous fixed-effect model for the study specific odds ratios. The Peto one-step estimator is based on quantities that are required for the calculation of the Mantel-Haenszel test. It is also used for meta-analysis of observational studies (Greenland and Salvan, 1990) and for estimating hazard ratio in a clinical trial (Berry, Kitchin and Mock, 1991), because the logrank test is the same as the Mantel-Haenszel test (Rothman and Greenland, 1998, p. 294).

Since the Peto one-step estimator is asymptotically unbiased under the null hypothesis that the common odds ratio is unity, one may expect its bias toward the null. However, Greenland and Salvan (1990) reported its substantial upward bias through a few numerical examples when the marginal totals are badly balanced. Table 1 shows an example given in Greenland and Salvan (1990). The odds ratio from the example in Table 1 is 3.75 . In contrast, the Peto one-step odds ratio is 8.37 .

Table 1: Data from a hypothetical cohort study (Study 3 in Table I, Greenland and Salvan, 1990)

\begin{tabular}{lccc}
\hline & Event & No Event & Total \\
\hline Treated & 3 & 18 & 21 \\
Control & 12 & 270 & 282 \\
\hline Total & 15 & 288 & 303 \\
\hline
\end{tabular}

\footnotetext{
* Department of Biostatistics, Kyoto University School of Public Health, Yoshida Konoe-cho, Sakyo-ku, Kyoto 606-8501, Japan. Tel +81-75-753-4475 shun@pbh.med.kyoto-u.ac.jp
} 
In this paper, the asymptotic expectation of the Peto one-step estimator is given. A numerical evaluation of its large sample bias is performed systematically for 1152 parameter combinations. The conventional variance of the Peto one-step estimator is also not consistent under non-null odds ratios. The true asymptotic variance and its estimator are also given and it is compared to the conventional variance estimator.

\section{Asymptotic Expectation and Variance of the Peto One-Step Estimator}

Consider a set of studies indexed by $k, k=1, \ldots, K$, and the data in study $k$ are summarized in a $2 \times 2$ table as follows:

\begin{tabular}{lccc}
\hline & Event & No Event & Total \\
\hline Treated & $x_{k}$ & $n_{k}-x_{k}$ & $n_{k}$ \\
Control & $y_{k}$ & $m_{k}-y_{k}$ & $m_{k}$ \\
\hline Total & $t_{k}$ & $N_{k}-t_{k}$ & $N_{k}$ \\
\hline
\end{tabular}

The Mantel-Haenszel test statistic for no association between treatment and event is given by

$$
X_{M H}^{2}=\frac{(X-E)^{2}}{V_{0}},
$$

where

$$
X=\sum_{k} x_{k}, \quad E=\sum_{k} \frac{n_{k} t_{k}}{N_{k}}, \quad V_{0}=\sum_{k} \frac{n_{k} m_{k} t_{k}\left(N_{k}-t_{k}\right)}{N_{k}^{2}\left(N_{k}-1\right)},
$$

and it has an approximate chi-squared distribution with 1 degree of freedom under the null hypothesis of no association between treatment and event.

Based on the product of the extended hypergeometric distributions, the conditional maximum likelihood estimator for the common log odds ratio, $\beta$, is calculated by Fisher's scoring method (McCullagh and Nelder, 1989, p. 42). The $i+1$ th iterated estimate of $\beta$ is given by

$$
\hat{\beta}^{i+1}=\hat{\beta}^{i}+\frac{X-\sum_{k} E\left(x_{k} \mid t_{k}, \hat{\beta}^{i}\right)}{\sum_{k} \operatorname{var}\left(x_{k} \mid t_{k}, \hat{\beta}^{i}\right)},
$$

where $E$ and $v a r$ are the exact expectation and variance of the extended hypergeometric distribution. When we start from the null value that $\beta^{0}=0$, we have a one-step improved estimator (Sato et al., 1998) as

$$
\hat{\beta}_{p}=\hat{\beta}^{1}=\frac{X-\sum_{k} E\left(x_{k} \mid t_{k}, \beta=0\right)}{\sum_{k} \operatorname{var}\left(x_{k} \mid t_{k}, \beta=0\right)} .
$$

This is the Peto one-step estimator. For the calculation of the confidence interval of the common log odds ratio, the null variance of the Peto one-step estimator, $1 / V_{0}$, is conventionally used.

To obtain the asymptotic expectation of the Peto one-step estimator, we consider the fixed studies limiting model in which the number $K$ of studies remains fixed but each $N_{k} \rightarrow \infty$ in such a way that $n_{k} / N_{k}$ and $N_{k} / N$ approach nonzero limits $l_{k}$ and $r_{k}$, where $N=\sum_{k} N_{k}$. Suppose the number of events $\left(x_{k}, y_{k}\right)$ are pairs of independent binomial observations with denominators $\left(n_{k}, m_{k}\right)$ and event probabilities $\left(p_{1 k}, p_{0 k}\right)$. Under the 
fixed studies limiting model, the common log odds ratio will have the same asymptotic distribution under both the extended hypergeometric and the two independent binomial distributions. The asymptotic expectation of the Peto one-step estimator is given by

$\beta_{p}=\frac{E^{A}(X-E)}{E^{A}\left(V_{0}\right)}=\frac{\sum_{k} r_{k} l_{k}\left(1-l_{k}\right)\left(p_{1 k}-p_{0 k}\right)}{\sum_{k} r_{k} l_{k}\left(1-l_{k}\right)\left[l_{k} p_{1 k}+\left(1-l_{k}\right) p_{0 k}\right]\left[l_{k}\left(1-p_{1 k}\right)+\left(1-l_{k}\right)\left(1-p_{0 k}\right)\right]}$,

where $E^{A}$ means the asymptotic expectation.

The conventional variance estimator, $1 / V_{0}$, is not consistent under the non-null common $\log$ odds ratio. The true asymptotic variance of the Peto one-step estimator is given by

$$
\begin{aligned}
\operatorname{Nvar}^{A}\left(\hat{\beta}_{p}\right) & =N \frac{\operatorname{var}^{A}(X-E)}{\left[E^{A}\left(V_{0}\right)\right]^{2}} \\
& =\frac{\sum_{k} r_{k} l_{k}\left(1-l_{k}\right)\left[\left(1-l_{k}\right) p_{1 k}\left(1-p_{1 k}\right)+l_{k} p_{0 k}\left(1-p_{0 k}\right)\right]}{\left(\sum_{k} r_{k} l_{k}\left(1-l_{k}\right)\left[l_{k} p_{1 k}+\left(1-l_{k}\right) p_{0 k}\right]\left[l_{k}\left(1-p_{1 k}\right)+\left(1-l_{k}\right)\left(1-p_{0 k}\right)\right]\right)^{2}},
\end{aligned}
$$

where $\operatorname{var}^{A}$ means the asymptotic variance. Hence a consistent estimator of the asymptotic variance of the Peto one-step estimator is obtained by

$$
V_{p}=\frac{\sum_{k} \frac{1}{N_{k}^{2}}\left[\frac{m_{k}^{2} x_{k}\left(n_{k}-x_{k}\right)}{n_{k}-1}+\frac{n_{k}^{2} y_{k}\left(m_{k}-y_{k}\right)}{m_{k}-1}\right]}{V_{0}^{2}}
$$

\section{Numerical Evaluation}

We performed a numerical evaluation for the asymptotic bias in the Peto one-step estimator. Table 2 shows selected parameter values: true common log odds ratio, $\beta$, was ranged from -1.61 to 1.61 ( 0.2 to 5 in the odds ratio scale, 12 levels); number of studies, $K$, from 5 to 40 (4 levels); ratio of treated subjects to control subjects from 1:1 to 1:10 (4 levels); the maximum of proportion of event in control group, $p_{0 k}$, was fixed at 0.2 , the minimum $\left(p_{\min }\right)$ was set to 0.01 to 0.1 (3 levels), and we examined equally spaced proportions of event in the control group that $p_{0 k}=p_{\min }+\left(0.2 p_{\min }\right)(k-1) /(K-1)$ and exponentially spaced ones that $p_{0 k}=p_{\min } \exp \left[\left(\log 0.2-\log p_{\min }\right)(k-1) /(K-1)\right]$ (2 levels). We evaluated total of 1152 parameter combinations.

Table 2: Selected parameter values for the bias evaluation

\begin{tabular}{ll}
\hline true common log odds ration, $\beta$ & $-1.61,-1.39,-1.11,-0.69,-0.41,-0.22$, \\
& $0.22,0.41,0.69,1.11,1.39,1.61$ \\
\hline number of studies, $K$ & $5,10,20,40$ \\
\hline ratio of number treated to control & $1: 1,1: 2,1: 5,1: 10$ \\
\hline proportion of event in control group, $p_{0 k}$ & maximum 0.2, minimum $(0.1,0.05,0.01)$ \\
\hline
\end{tabular}




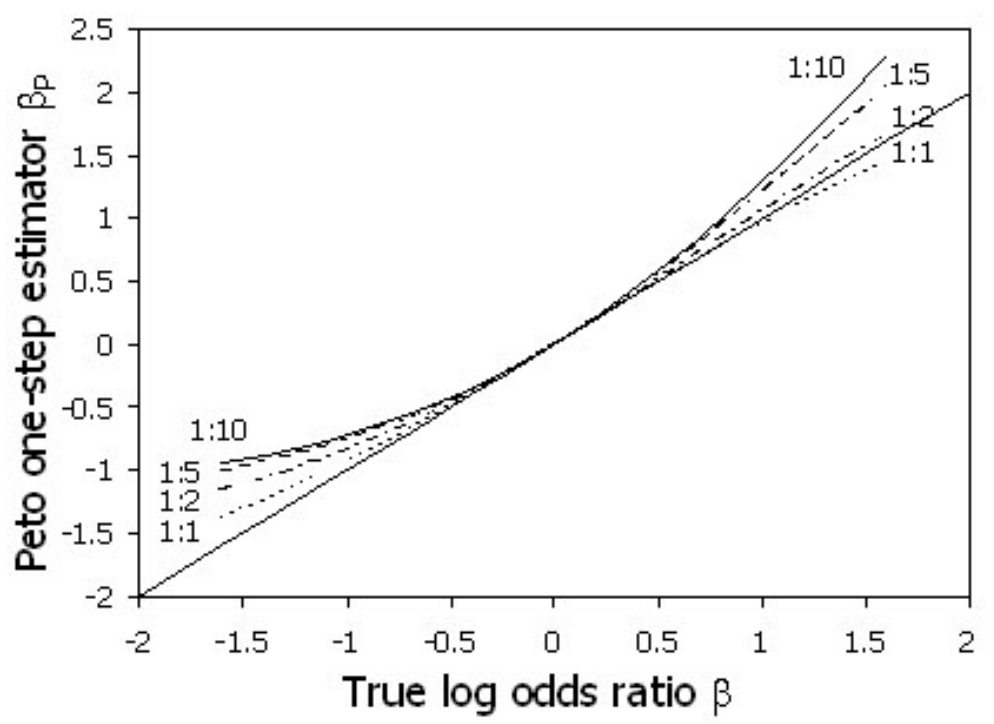

Figure 1. Bias in the Peto one-step estimator $K=20 ; p_{0 k}=0.05-0.2$, exponentially spaced

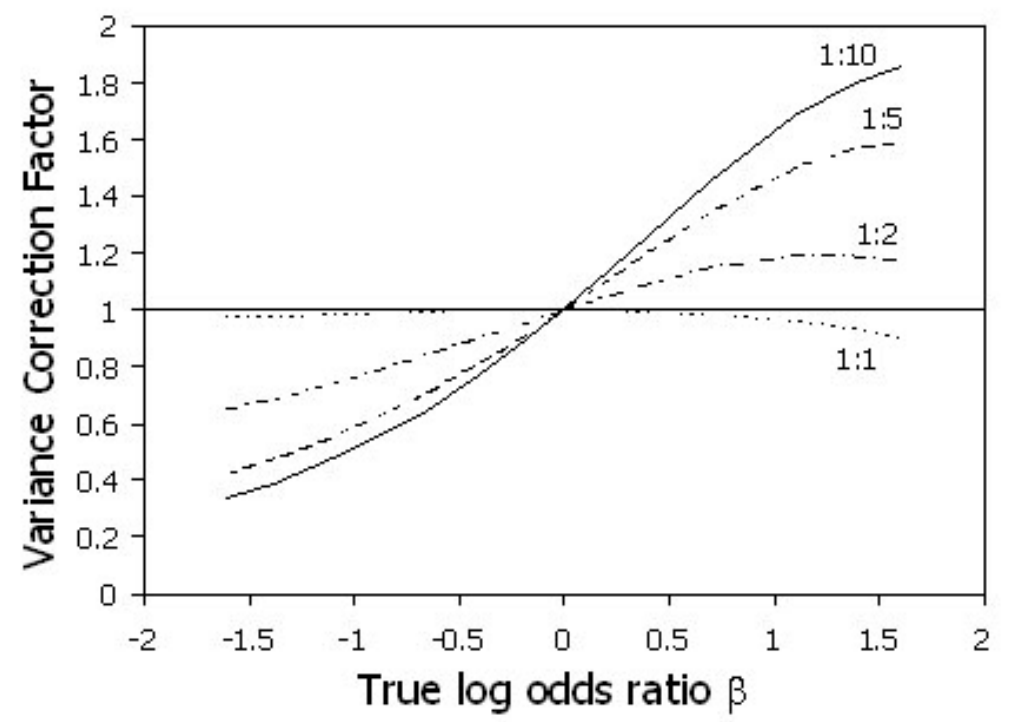

Figure 2. Variance correction factor to the conventional variance $K=20 ; p_{0 k}=0.05-0.2$, exponentially spaced

An illustration of the magnitude of bias where $K=20$ and $p_{0 k}=0.05-0.2$ (exponentially spaced) is shown in Fig. 1. When the ratio of treated subjects to control subjects was 1:1, the bias in the Peto one-step estimator was toward the null. When the 
true log odds ratio was less than 0 , its bias was also toward the null. However, when the treated-control ratio was greater than 1 and the true log odds ratio was greater than 0 , the bias tended to be anti-conservative. In general, the upward bias tended to be severe with increase of $\beta(>0)$ and treated-control ratio.

We also examined the variance correction factor which was the ratio of the true asymptotic variance of the Peto one-step estimator to the asymptotic expectation of its conventional variance. We used the same parameter combinations in Table 1. Fig. 2 shows an illustration of the results for the same conditions as in Fig. 1. When the treated-control ratio was $1: 1$, the conventional variance was virtually unbiased. However, with increase of treated-control ratio, the conventional variance was biased upward (correction factors were less than 1 ) when $\beta<0$ and was biased downward (correction factors were greater than 1) when $\beta>0$.

\section{Discussion}

As shown in Fig. 1, the bias in the Peto one-step estimator was small and toward the null when the treated-control ratio was $1: 1$. Since the bias in its conventional variance was also small (Fig. 2), one may use the Peto one-step estimator and associated confidence intervals when the treated-control ratio is 1:1 which is typical in the meta-analysis of clinical trials.

However, when the treated-control ratio is greater than 1:1 as in the meta-analysis of observational studies, the biases in the Peto one-step estimator and its conventional variance become severe, especially when the treatment has a large effect. In such a case, one can use the Mantel-Haenszel methods and their associated confidence intervals, instead of the Peto one-step method or the odds ratio as a measure of treatment effect (Greenland and Robins, 1985; Robins, Breslow, and Greenland, 1986; Sato 1989, 1990).

\section{References}

Berry, G., Kitchin, R.M. and Mock P.A. (1991). A comparison of two simple hazard ratio estimators based on the logrank test, Statistics in Medicine, 10, 749-755.

Greenland, S. and Robins, J.M. (1985). Estimation of a common effect parameter from sparse follow-up data, Biometrics 41, 55-68.

Greenland, S. and Salvan A. (1990). Bias in the one-step method for pooling study results, Statistics in Medicine 9, 247-252.

McCullagh, P. and Nelder, J.A. (1989). Generalized Linear Models, 2nd ed., Chapman and Hall, London.

Mosteller, F. and Chalmers, T.C. (1992). Some progress and problems in meta-analysis of clinical trials, Statistical Science 7, 227-236.

Petitti, D.B. (1994). Meta-Analysis, Decision Analysis and Cost-Effectiveness Analysis, Oxford University Press, New York.

Robins, J.M., Breslow, N.E. and Greenland, S. (1986). Estimation of the MantelHaenszel variance consistent in both sparse data and large-strata limiting model, Biometrics 42, 311-323. 
Rothman K.J. and Greenland, S. (1998). Modern Epidemiology, 2nd ed., LippincottRaven, Philadelphia.

Sato, T. (1989). On the variance estimator for the Mantel-Haenszel risk difference (letter), Biometrics 45, 1323-1324.

Sato, T. (1990). Confidence limits for the common odds ratio based on the asymptotic distribution of the Mantel-Haenszel estimator, Biometrics 46, 71-80.

Sato, T., Takagi, H., Yanagawa, T. and Yanagimoto, T. (1998). The Mantel-Haenszel method for stratified analysis of $2 \times 2$ tables, Proceedings of the Institute of the Statistical Mathematics 46, 153-177 (in Japanese).

Yusuf, S., Peto, R., Lewis, J., Collins, R. and Sleight, P. (1985). Beta blockade during and after myocardial infarction: an overview of the randomized trials, Progress in Cardiovascular Diseases 27, 335-371.

Received October 28, 2003

Revised April 8, 2004 